\title{
Role of Pharmacoeconomics in Precision Medicine
}

\author{
Zahra Ghasemi (iD ${ }^{1,}$, , Saeid Safari ${ }^{2}$ and Alireza Zali ${ }^{2}$ \\ ${ }^{1}$ Department of Pharmacoeconomics and Pharma Management, School of Pharmacy, Shahid Beheshti University of Medical Sciences, Tehran, Iran \\ ${ }^{2}$ Functional Neurosurgery Research Center, Shohada Tajrish Comprehensive Neurosurgical Center of Excellence, Shahid Beheshti University of Medical Sciences, Tehran, Iran \\ "Corresponding author: Department of Pharmacoeconomics and Pharma Management, School of Pharmacy, Shahid Beheshti University of Medical Sciences, Tehran, Iran. \\ Email: matinaghasemi@gmail.com
}

Received 2021 June 07; Revised 2021 June 23; Accepted 2021 July 11.

Keywords: Precision Medicine, Personalized Diagnostic Tests, Pharmacoeconomics, Health Technology Assessment, Cost-Effectiveness Analysis

Precision medicine (PM) and all related concepts, like personalized medicine and stratified medicine, are characterized by a growing number of diagnostic tests for targeting healthcare interventions and choosing the most proper treatment for each patient. This part of medical knowledge is highlighted as an essential program for national health policies. The authentic explanation of precision or personalized medicine from the perspective of the Council of Advisors on Science and Technology in 2008 is as follows:

"The accommodating of options for treating the individuals based on their characteristics to categorize them to sub-group of patients with different susceptibility to a special disease or their body response to a distinct treatment. Preventative or therapeutic interventions can then be fixed on those who will benefit, saving monetary resources and preventing adverse effects for those who will not (1)."

There are three types of precision medicine applications: disease susceptibility prediction, patients' prognosis, and treatment response or side effect prediction (2). Predicting a patient's vulnerability to disease is essential, mainly in cancerous patients. For example, patients with a mutation in BRCA1/2 can benefit from genomic screening to determine their breast cancer-related risks (3).

Decision-makers often dispute over improving clinical outcomes and more effectively using healthcare resources via personalizing the treatment (4). Thus, the main barrier in the implementation of this program is determining payers and reimbursement organizations. There is a great need from decision-makers' perspectives to have a proper tool to make better decisions for resource allocation and cost-saving of targeting therapy, which could be feasible through conducting economic evaluation (5).
A model of economic evaluation depends on a systematic assortment of data from so many sources within a welldefined framework, like evidence of clinical effectiveness, utility and cost information extracted from the literature or practice-based data, and opinions from relevant experts. The "gold standard" for the assortment of data for economic models is through systematic reviews of published literatures. Pharmacoeconomic studies have provided a comparative analysis of clinical outcomes and costs of using those technologies for decision-makers to make proper health budget allocation decisions (6).

The definite objections in modeling and conducting health economic evaluation methods in precision medicine are centered on medicine assessment and pharmacogenetic technologies via systematic reviews. The frequent use of cost-effectiveness analyses in developing precision medicine strategies in cancer treatment is expected to enhance the implementation of such strategies in routine practice. However, it needs to be assessed how costeffectiveness studies can be systematically applied during the development process of new technologies (7).

Among the four types of analysis in health economic evaluation, cost-utility analysis (CUA) and costeffectiveness analysis (CEA) are most frequently used; among performed studies in this category, had evaluated clinical molecular genetic tests. In studies using CUA, quality-adjusted life-years (QALY) is the metric of health outcomes, and in those using CEA, the incremental costeffectiveness ratio is the measure to show the amount of benefit derived by making an alternative treatment choice (8).

In models used in this area, differences in the target population and test purposes lead to six types of tests: (1)

Copyright (c) 2021, Precision Medicine and Clinical OMICS. This is an open-access article distributed under the terms of the Creative Commons Attribution-NonCommercial 4.0 International License (http://creativecommons.org/licenses/by-nc/4.0/) which permits copy and redistribute the material just in noncommercial usages, provided the original work is properly cited. 
screening,(2) diagnostic, (3) predictive, (4) surveillance,(5) prognostic, and (6) monitoring.

New screening tests are helpful as they increase life expectancy and QALY by early determining the risks of disease, reducing costs related to unnecessary tests, and improving patients' compliance by choosing the most proper treatment for them. Here, we briefly described benefits of each of the tests:

Diagnostic tests can improve the quality of life by reducing false-negative tests and unnecessary treatment costs.

Predictive tests can improve the response rate to treatments and increase life expectancy and the quality of life by determining specific treatment and reducing the rate of toxicity of adverse events (9).

Surveillance tests can increase the quality and expectancy of life by early identification of disease recurrence and effective interventions for its treatment. These tests also reduce costs and increase the quality of life by reducing unnecessary tests and treatment.

Finally, prognostic tests improve the response rate and reduce treatment costs for patients (10). All the mentioned pathways are briefly described in Figure 1.

This study aimed to define the actual objective in the cost-effectiveness analysis of precision medicine and improve related methodologies. Many factors affect the costeffectiveness of precision medicine, such as the prevalence of the genetic status (some allele or mutation) in a specific population, the high price of genetic tests and associated treatment, the probability of mortality or any complication, the health-related quality of life before and after a preventive medicine, the diagnosis time of a risk factor, and patient adherence to treatment. It appears that despite the high costs of conducting precision tests and therapy, they still need to be implemented because of the great value of the PM use. However, many challenges exist for economic evaluation methods in PM due to significant limitations of clinical trials. Accordingly, data in these trials are usually extrapolated to populate the economic model, which may not be transformable to real-world settings that are genetically different. However, to ensure the value and benefits of conducting PM with all the hurdles, many studies around the world have been published on evaluations of pharmacogenetic tests. In contrast, other studies have focused more on the PM nature. In general, these studies mention that the number of economic evaluations of precision medicine is growing daily. However, there is a kind of limited evidence on the value of economic parts of precision medicine, and many gaps in topics discussed.

There have been many literatures on the cost- effectiveness of conducting precision medicine and individualizing treatment since 2012, and most of them have shown that implementing different screening tests can reduce costs of the healthcare system, reduce adverse drug reaction incidence and its management cost, and improve reimbursement condition, increase the quality and expectancy of life.

\section{Footnotes}

Authors' Contribution: Study design, Zahra Ghasemi; Drafting the interpretation, Zahra Ghasemi; Critical revision of the manuscript for important intellectual content, Zahra Ghasemi; Data support, Zahra Ghasemi.

Conflict of Interests: None to declare.

\section{References}

1. Ginsburg GS, Phillips KA. Precision medicine: From science to value Health Aff(Millwood).2018;37(5):694-701. doi:10.1377/hlthaff.2017.1624. [PubMed: 29733705]. [PubMed Central: PMC5989714].

2. Payne K, Eden M, Davison N, Bakker E. Toward health technology assessment of whole-genome sequencing diagnostic tests: Challenges and solutions. Per Med. 2017;14(3):235-47. doi: 10.2217/pme-2016-0089. [PubMed: 29767583].

3. Bertier G, Carrot-Zhang J, Ragoussis V, Joly Y. Integrating precision cancer medicine into healthcare-policy, practice, and research challenges. Genome Med. 2016;8(1):108. doi: 10.1186/s13073-016-0362-4. [PubMed: 27776531]. [PubMed Central: PMC5075982].

4. Kasztura M, Richard A, Bempong NE, Loncar D, Flahault A. Costeffectiveness of precision medicine: A scoping review. Int J Public Health. 2019;64(9):1261-71. doi: 10.1007/s00038-019-01298-x. [PubMed: 31650223]. [PubMed Central: PMC6867980].

5. Shabaruddin FH, Fleeman ND, Payne K. Economic evaluations of personalized medicine: Existing challenges and current developments. Pharmgenomics Pers Med. 2015;8:115-26. doi: 10.2147/PGPM.S35063. [PubMed: 26309416]. [PubMed Central: PMC4538689].

6. Gavan SP, Thompson AJ, Payne K. The economic case for precision medicine. Expert Rev Precis Med Drug Dev. 2018;3(1):1-9. doi: 10.1080/23808993.2018.1421858. [PubMed: 29682615]. [PubMed Central: PMC5890303]

7. Grosse SD. Economic analyses of genetic tests in personalized medicine: clinical utility first, then cost utility. Genet Med. 2014;16(3):225-7. doi: 10.1038/gim.2013.158. [PubMed: 24232411] [PubMed Central: PMC4454889].

8. Palmer S, Byford S, Raftery J. Economics notes: Types of economic evaluation. Bmj. 1999;318(7194):1349. doi: 10.1136/bmj.318.7194.1349.

9. Sroczynski G, Schnell-Inderst P, Muhlberger N, Lang K, Aidelsburger $\mathrm{P}$, Wasem J, et al. Cost-effectiveness of primary HPV screening for cervical cancer in Germany-a decision analysis. Eur J Cancer. 2011;47(11):1633-46. doi:10.1016/j.ejca.2011.03.006. [PubMed: 21482103].

10. Horster L, Schlenk RF, Stadler M, Gabriel M, Thol F, Schildmann J, et al. Cost-effectiveness of methods in personalized medicine. Results of a decision-analytic model in patients with acute myeloid leukemia with normal karyotype. Leuk Res. 2017;62:84-90. doi 10.1016/j.leukres.2017.09.009. [PubMed: 28988037]. 
Ghasemi Z et al.

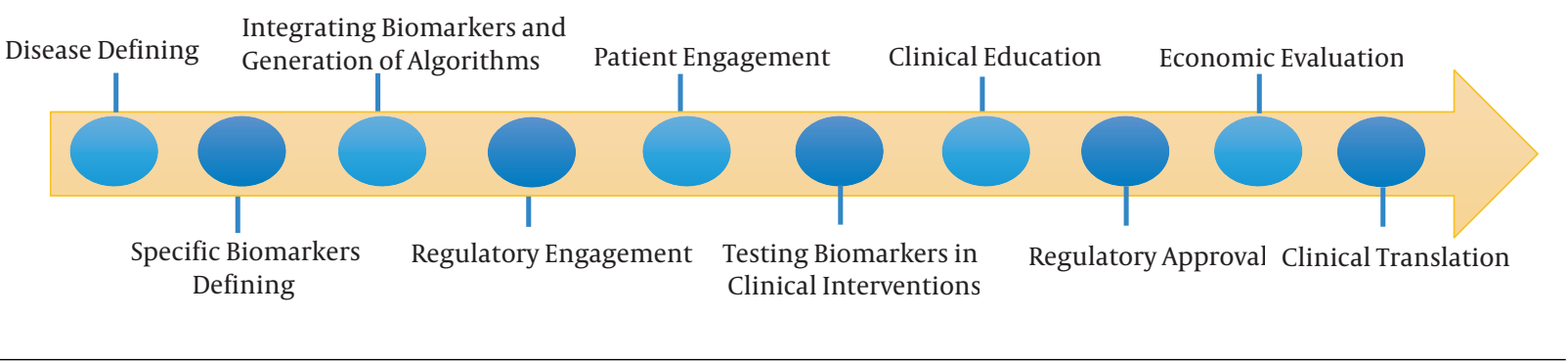

Figure 1. Pathway of diagnosis and evaluation in precision medicine

Precis Med Clin OMICS. 2021; 1(1):e115774.

3 